\title{
Efficient Zero-Knowledge Proofs
}

\author{
Jens Groth \\ UCL, London, UK
}

\begin{abstract}
A zero-knowledge proof is a two-party protocol that enables a prover to convince a verifier of the truth of a statement without revealing anything else. Zero-knowledge proofs are widely used in cryptography to guarantee that parties are acting correctly without revealing their private information.

Interestingly, it is possible to make highly efficient zero-knowledge proofs where the amount of communication is much smaller than the size of the statement. We will in this talk discuss practical communicationefficient zero-knowledge proofs.
\end{abstract}

